\title{
LUXAÇÃO TRAUMÁTICA POSTERIOR DO QUADRIL EM CRIANÇAS: RELATO DE CINCO CASOS
}

\author{
TRAUMATIC POSTERIOR DISLOCATION OF THE HIP IN CHILDREN: REPORT OF FIVE CASES
}

\begin{abstract}
Gilberto Francisco Brandão', Luiz Renato Drumond Américo², Cláudio Beling Gonçalves Soares³, Rodrigo Galinari Costa Faria ${ }^{4}$, Luiz Eduardo Moreira Teixeira ${ }^{5}$
\end{abstract}

\section{RESUMO}

Objetivo: Avaliar uma série de casos de luxação traumática posterior em crianças, o tratamento e os resultados, e revisar os aspectos relacionados à sua epidemiologia, diagnóstico clínico e radiográfico, tratamento, complicações e prognóstico. Métodos: Foram avaliados retrospectivamente cinco pacientes com luxação traumática do quadril com média idade de 4,6 \pm 0,9 anos e tempo de seguimento de 19,8 \pm 7,0 meses. Foram avaliados o tempo entre a luxação e a redução, o tipo de tratamento, as lesões associadas e as complicações tardias. Resultados: O tratamento inicial foi a redução incruenta com tempo médio de 5,2 \pm 3,6 horas após o trauma inicial, sendo que todos foram submetidos à redução sob anestesia. O tratamento complementar incluiu imobilização gessada e tração. Não foi observada necessidade de cirurgias adicionais ou sequelas a longo prazo. Conclusão: A luxação traumática do quadril deve ser tratada com redução incruenta rápida, controle adequado da redução e observação rigorosa para diagnóstico e tratamento de complicações tardias.

Descritores - Luxação do quadril/epidemiologia; Luxação do quadril/diagnóstico; Luxação do quadril/radiografia; Luxação do quadril/complicações; Criança

\section{ABSTRACT}

Objective: To evaluate a series of cases of traumatic posterior dislocations in children, the treatment and the results, and to revise aspects such as the epidemiology, clinical and radiographic diagnosis, treatment, complications and prognosis. Methods: Five patients with traumatic hip dislocation, with an average age of 4.6 \pm 0.9 years, and an ongoing follow-up period of $19.8 \pm 7.0$ months, were evaluated retrospectively. The time between dislocation and reduction, the type of treatment, associated injuries, and subsequent complications were also evaluated. Results: The initial treatment was closed reduction, in an average time of $5.2 \pm 3.6$ hours after the initial trauma, in which the patients were subjected to the reduction under anesthesia. Complementary treatment included immobilization with casts and traction. No needs for additional surgeries or long-term side effects were observed. Conclusion: Traumatic dislocation of the hip should be treated by quick closed reduction, with appropriate control of the reduction and careful observation of the patient for diagnosis and treatment of subsequent complications.

Keywords: Hip dislocation/epidemiology; Hip dislocation/ diagnosis; Hip dislocation/radiography; Hip dislocation/complications; Child

complicações. Entretanto, o tratamento após a redução permanece sem consenso ${ }^{(1)}$.

As complicações precoces mais comuns são as fraturas associadas e a lesão neurológica. Complicações tardias incluem episódios de reluxação, condrólise, necrose avascular e a artrite degenerativa, usualmente secundárias ao atraso da redução, sendo fatores relacionados ao pior prognóstico: a permanência da luxação por mais de seis horas, a maturidade esquelética avançada, a lesão articular grave e a presença de traumas múltiplos associados ${ }^{(1)}$.

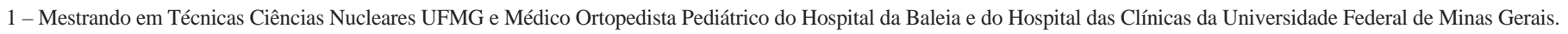

2 - Médico Ortopedista do Hospital da Baleia.

3 - Médico Ortopedista Pediátrico do Hospital da Baleia.

4 - Médico Ortopedista do Hospital Infantil São Camilo.

5 - Doutorando em Cirurgia e Professor do Departamento do Aparelho Locomotor da Universidade Federal de Minas Gerais.

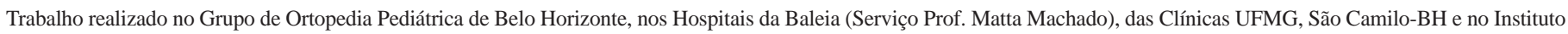
Mineiro de Ortopedia e Traumatologia.

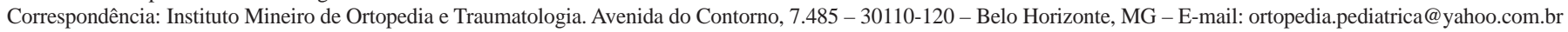


O objetivo deste estudo é avaliar uma série de casos de luxação traumática posterior em crianças, o tratamento e os resultados, e revisar os aspectos relacionados à sua epidemiologia, diagnóstico clínico e radiográfico, tratamento, complicações e prognóstico.

\section{PACIENTES E MÉTODO}

Foram avaliados cinco casos de luxação traumática posterior do quadril em pacientes com esqueleto imaturo no período de setembro de 1999 a dezembro de 2003 atendidos nos Hospitais da Baleia e São Camilo, e no Instituto Mineiro de Ortopedia. A média idade foi de 4,6 $\pm 0,9$ anos (variando de 3,9 a seis anos). Quatro (80\%) crianças eram do sexo masculino e uma (20\%) era do sexo feminino. O mecanismo de lesão foi trauma direto em um (20\%), queda da própria altura em três (60\%) e queda de laje em um (20\%), o lado acometido foi o esquerdo em quatro pacientes (80\%) e direito em um (20\%).

Em todos os casos o diagnóstico foi baseado no exame físico e nos achados radiográficos do quadril em anteroposterior. As cinco crianças apresentavam ao exame incapacidade para marcha e deformidade típica em flexão, adução e rotação medial do quadril acometido (Figuras 1,2A). Todos foram submetidos à redução incruenta sob anestesia venosa e o controle pósredução foi realizado com radiografias convencionais (Figura 2B) e tomografia computadorizada na suspeita de incongruência articular e possíveis lesões ocultas. O acompanhamento era realizado semanalmente nos dois primeiros meses, mensalmente nos dois primeiros anos e semestral a partir do terceiro anos de redução. O controle consistia de avaliação clínica da amplitude de movimen-

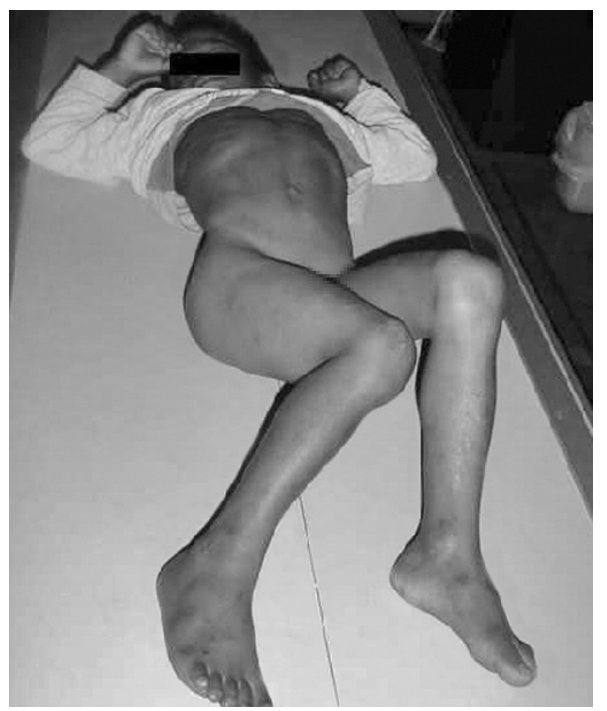

Figura 1 - Aspecto clínico característico da luxação posterior do quadril

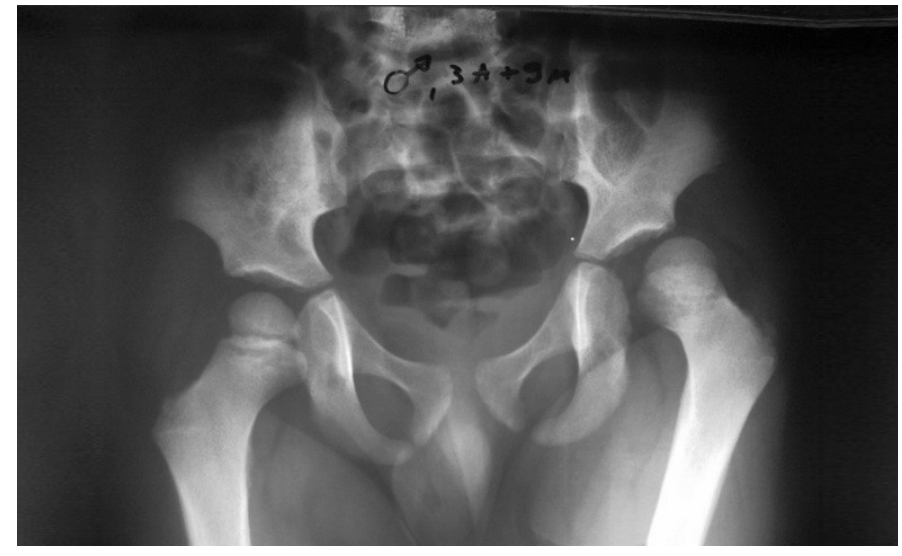

Figura 2A - Aspecto radiográfico de luxação traumática posterior do quadril

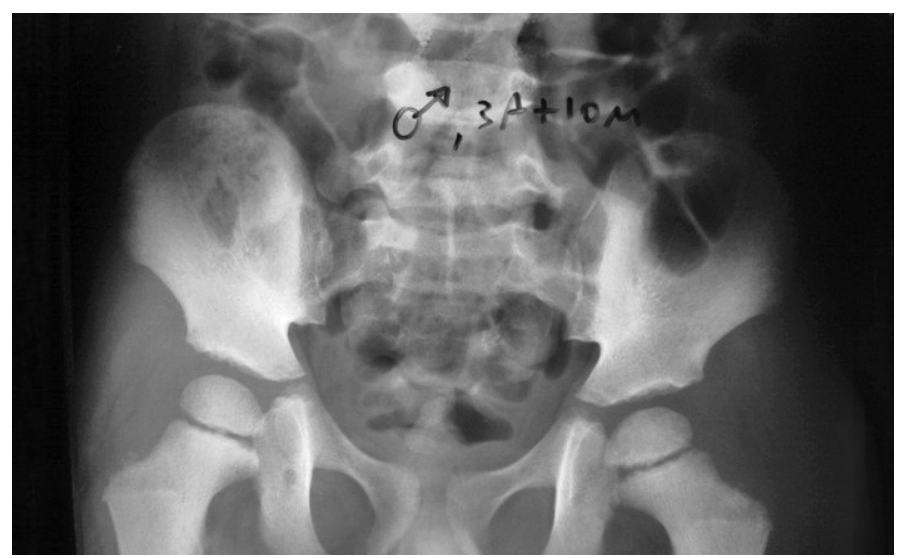

Figura 2B - Após redução da luxação e sem sinais de necrose após sete meses

to, padrão da marcha, discrepância de membros e da avaliação radiográfica em anteroposterior e batráquio. O tempo médio de seguimento foi de 19,8 \pm 7,0 meses. Foram avaliados: a intensidade do trauma, o tempo inicial entre o trauma e a redução, as lesões associadas, o resultado funcional através da limitação da mobilidade articular e claudicação residual, o resultado radiográfico através da análise da esfericidade da cabeça femoral e de alterações no desenvolvimento acetabular e novos episódios de luxação. Os dados foram analisados com auxílio do software Epi-Info versão 6.04.

O trabalho foi aprovado pelo Comitê de Ética dos respectivos Hospitais e consentimento dos pais responsáveis através do Termo de Consentimento Livre e Informado.

\section{RESULTADOS}

O trauma que resultou na luxação foi de baixa energia em quatro (80\%), sendo apenas um de alta energia por queda de laje, (aproximadamente 2,5m) (Tabela 1). O tratamento inicial foi redução incruenta sob anestesia com tempo médio de 5,2 \pm 3,6 horas após o trauma 
inicial (mediana de 3,5 horas). Epifisiólise do fêmur proximal foi observada em um caso, sendo que após a redução, os exames radiográfico e tomográfico demonstraram redução espontânea que permaneceu estável no controle radioscópico, optando-se por tratamento conservador com gesso pelvipodálico. Nenhum dos casos apresentou alterações neurológicas como complicação. No estudo pós-redução, foi observada congruência articular em todos os casos não havendo interposição ou lesões associadas ocultas ao exame radiográfico.

Tabela 1 - Tratamento complementar após redução incruenta dos pacientes com luxação posterior do quadril

\begin{tabular}{c|c|c|c|c}
\hline Paciente & Gênero & $\begin{array}{c}\text { Tratamento } \\
\text { complementar }\end{array}$ & $\begin{array}{c}\text { Tempo de } \\
\text { imobilização } \\
\text { (semanas) }\end{array}$ & $\begin{array}{c}\text { Tempo para } \\
\text { descarga de peso } \\
\text { (semanas) }\end{array}$ \\
\hline DBL & M & GPP & 3 & 4 \\
\hline FFL & F & GPP & 4 & 8 \\
\hline ASR & F & TTE + GPP & 4 & 4 \\
\hline BCMM & F & TTE + GPP & 4 & 4 \\
\hline EML & F & GPP & 4 & 4 \\
\hline
\end{tabular}

Fonte: SAME - Hospital da Baleia, Hospital São Camilo e IMOT

Legendas: GPP - gesso pelvipodálico, TTE - tração esquelética, M - masculino, F - feminino

Após o tratamento complementar, todas as crianças foram liberadas para marcha sem restrições. Durante o acompanhamento não foram observadas alterações clínicas ou episódios de reluxação, sendo que todos os pacientes apresentavam amplitude de movimento simétrica ao quadril contralateral após três meses do trauma e não apresentaram claudicação residual. A evolução radiográfica foi satisfatória, com manutenção da esfericidade da cabeça femoral, da orientação acetabular e da congruência articular. Condrólise, necrose avascular (NAV) ou degeneração articular não foram observadas durante seguimento. Em nenhum dos casos houve necessidade de tratamento cirúrgico complementar.

\section{DISCUSSÃO}

A luxação traumática do quadril em crianças (LTQC) é o deslocamento traumático de uma articulação normal, em pacientes com menos de 16 anos de idade ${ }^{(2)}$. É uma lesão rara, com incidência de 0,8 casos por milhão/ano, sendo a luxação posterior correspondente a $80 \%$ dos $\operatorname{casos}^{(2-11)}$. É mais comum em meninos $(4: 1)^{(8,9)}$ e pode ocorrer em qualquer faixa etária, embora os picos de incidência sejam entre quatro e sete anos e entre 11 e $15 \operatorname{anos}^{(3,8,9)}$.

Nas crianças mais jovens, o acetábulo é muito flexível, frouxo e cartilaginoso ${ }^{(1-4)}$ permitindo que traumas triviais resultem em luxação ${ }^{(1,3-5,7-10,12)}$. Com o cresci- mento, a cartilagem se calcifica e a frouxidão ligamentar diminui, sendo necessário maior energia para deslocar o quadril $^{(1,2,10-12)}$. Assim, associação de LTQC com fratura femoral sempre deve ser considerada ${ }^{(11)}$, especialmente nas crianças maiores e adolescentes ${ }^{(1-4,10)}$.

Os casos descritos apresentam características semelhantes aos da literatura. Observamos predomínio do sexo masculino, faixa etária média de 4,6 anos e trauma de menor energia na maioria dos casos, com exceção de um paciente que sofreu queda de altura e que apresentou epifisiólise proximal do fêmur associada.

O diagnóstico clínico desta lesão se baseia na história do trauma, na dor no quadril e na incapacidade de marcha $^{(2)}$. A deformidade típica varia de acordo com o tipo de luxação. Na luxação posterior o membro inferior estará fletido, aduzido e em rotação interna ${ }^{(1-4,9)}$. A função do nervo ciático deve ser registrada antes e após a redução do quadril ${ }^{(3,4)}$. Há também possibilidade de redução espontânea, mas parcial da luxação, por interposição de tecidos moles ${ }^{(13)}$. Esta situação pode não ser reconhecida, levando ao dano articular permanente $e^{(1,12,14,15)}$.

A avaliação radiográfica de boa qualidade é essencial para confirmar o diagnóstico, revelar o tipo de luxação e descartar fraturas associadas ${ }^{(2)}$. Frequentemente, fragmentos da fratura do acetábulo ou do fêmur são mais bem visualizados nas radiografias antes da redução ${ }^{(3,4)}$. No nosso caso de fratura femoral a lesão era evidente apenas na radiografia inicial, em concordância com Canale ${ }^{(3)}$.

A LTQC é uma emergência ortopédica ${ }^{(7,11)}$. Há consenso que a redução deva ser imediata, preferencialmente fechada e sob anestesia geral ${ }^{(9,16,17)}$ ou com relaxantes ${ }^{(3,11)}$, utilizando as mesmas manobras de redução dos adultos (Stimson, Allis e Bigelow) $)^{(2,3,9,10)}$. Após a redução, devese avaliar a congruência articular, comparando o espaço articular, a lateralização da cabeça ${ }^{(14)}$ e a quebra na linha de Shenton ${ }^{(9)}$ com o lado contralateral.

Nos casos de redução incongruente, a tomografia computadorizada (TC) e a ressonância magnética (RM) são úteis para determinar a presença de fragmentos ou tecido interposto ${ }^{(14)}$. Se confirmada a interposição, realiza-se uma segunda tentativa de redução ${ }^{(3,4)}$ ou redução aberta por abordagem posterior ${ }^{(3,4,10)}$. As indicações para redução aberta são o fracasso na redução fechada, lesão do nervo ciático com indicação de exploração ${ }^{(2,3)}$ e fratura acetabular, do colo ou da cabeça femoral que exijam tratamento cirúrgico ${ }^{(2)}$.

O tratamento após a redução permanece sem consenso $^{(1)}$. Price et al $^{(12)}$ e Tachdjian ${ }^{(9)}$ recomendam gesso pelvipodálico (GPP) por quatro a seis semanas, para permitir cicatrização da cápsula. Gianom et $a l^{(6)}$ indicam 
repouso no leito até alívio da dor, seguido de marcha com muletas e proteção do apoio por quatro semanas. Blaster e Hughes ${ }^{(10)}$ propõem repouso até melhora da dor, seguido de retorno à marcha. Canale $\mathrm{e}^{(3)}$ e Hebert $^{(2)}$ utilizam tração cutânea durante uma semana, seguido de proteção do apoio por quatro a seis semanas. Quando há necessidade de redução aberta recomenda-se tração esquelética (TTE) ou GPP por até seis semanas ${ }^{(2,3,10)}$ e nas luxações com mais de 24 horas de evolução a TTE prévia à redução seguida de tração por duas a três semanas após a redução ${ }^{(2,3)}$.

Os cincos casos analisados foram submetidos à redução incruenta sob anestesia. A avaliação radiográfica da congruência articular foi realizada em todos eles. Na luxação associada a fratura femoral, a congruência foi confirmada por TC.

Muitas complicações estão associadas à LTQC. As lesões nervosas têm incidência aproximada de 5\% em crianças $^{(2,9)}$, sendo o ramo fibular do nervo ciático o mais atingido nas luxações posteriores por compressão direta ${ }^{(13)}$. A ausência de melhora em quatro a seis semanas indica exploração cirúrgica após estudos neurodiagnósticos ${ }^{(9)}$. Se há perda da função do ciático após a redução, o nervo deve ser explorado cirurgicamente ${ }^{(10)}$. A necrose avascular (NAV) tem incidência de $8 \%$ a $10 \%$, sendo o atraso na redução traumas de grande energia e crianças maiores de cinco anos fatores associados a maior frequência desta complicação ${ }^{(1-3,7)}$. O principal fator parece ser o tempo de luxação. A redução após seis horas de evolução aumenta em 20 vezes o risco de $\mathrm{NAV}^{(18)}$. Para Mehlman et $a l^{(18)}$ a avaliação cintilográfica não é necessária como rotina no acompanhamento. Para Blaster e Hughes ${ }^{(10)}$ é recomendável que o quadril seja avaliado por radiografias seriadas, por pelo menos dois anos após a luxação. Estes autores também não recomendam avaliação cintilográfica e por RM como rotina, com controle até a maturidade esquelética ${ }^{(19)}$. A reluxação é rara e associada a crianças abaixo de oito anos ${ }^{(10)}$ e crianças com frouxidão ligamentar, especialmente a síndrome de Down ${ }^{(3,10)}$. Seu tratamento consiste em nova redução seguida de GPP por seis semanas ou capsuloplastia ${ }^{(2,10)}$. Em crianças mais velhas pode ser necessário associar procedimentos ósseos como cirurgia de Salter ${ }^{(3,10)}$ ou osteotomia varizante ${ }^{(3)}$. A condrólise tem sido relatada após luxação traumática do quadril em $6 \%$ das crianças. Provavelmente é resultado da lesão articular no momento da luxação. O tratamento deve ser sintomático. Se não ocorrer a reconstituição articular, artrodese ou reconstrução deverá ser considerada ${ }^{(10)}$. Coxa magna parece ocorrer como resultado da hiperemia pós-traumática. $\mathrm{Na}$ maioria das crianças, esta condição é assintomática e não requer qualquer tratamento. A miosite ossificante ${ }^{(3)}$ e artrite degenerativa $^{(1,7-9)}$ são sequelas potenciais.

Os cinco casos relatados não apresentaram complicações até a última avaliação ortopédica. A maioria das crianças tem boa evolução a longo prazo e melhores resultados quando comparados aos adultos ${ }^{(10,20)}$. Uma grande percentagem de pacientes (78\%) atividades de alta demanda como futebol ou basquete ${ }^{(18)}$.

\section{CONCLUSÃO}

A LPQC, em nossa série de casos, apresentou-se em crianças mais jovens, com trauma de menor energia e com boa evolução tardia. Apesar da evolução satisfatória dos nossos cinco pacientes, o acompanhamento a longo prazo deve ser mantido.

\section{REFERÊNCIAS}

1. Kutty S, Thornes B, Curtin WA, Gilmore MF. Traumatic posterior dislocation of hip in children. Pediatr Emerg Care. 2001;17(1):32-5.

2. Hebert S. Fraturas e luxações do quadril na criança e no adolescente. In: Hebert S, Xavier R, Pardini Júnior AG, Barros Filho TEP. Ortopedia e Traumatologia - Princípios e Prática. 3a. ed., Porto Alegre: Artmed; 2003. P.1231-7.

3. Canale ST. Luxações traumáticas do quadril em crianças. In: Crenshaw AH. Cirurgia Ortopédica de Campbell. 8a. ed. São Paulo: Manole; 1996. p.1222-5.

4. Canale ST, King RE. Luxações traumáticas do quadril. In: Rockwood CA Jr, Wilkins KE, King RE. Fraturas em crianças. Tradução de Vilma Ribeiro de Souza Varga. 3a. ed. São Paulo: Manole; 1993. p.1061-89.

5. Guarniero R, Peixinho M. Luxação traumática do quadril na criança. Rev Bras Ortop. 1990; 25(4):93-6.

6. Gianom D, Kronberger G, Sacher P. Long-term follow-up of traumatic hip dislocation in childhood. Helv Chir Acta.1994;60(4):623-7.

7. Hughes MJ, D'Agostino J. Posterior hip dislocation in a five-year-old boy: a case report, review of the literature and current recommendations. J Emerg Med. 1996; 14(5):585-90.

8. Petrie SG, Harris MB, Willis RB. Traumatic hip dislocation during childhood. A case report and review of the literature. Am J Orthop. 1996;25(9):645-9.

9. Tachdjian MO. Luxação traumática do quadril. In: Tachdjian MO. Ortopedia pediátrica. Tradução de José Aparecido Lopes. 2a. ed. São Paulo: Manole; 1995. p. 3222-40.

10. Blaster RD, Hughes LO. Fractures and traumatic dislocation of the hip in children. In: Beaty JH, Kasser JR. Rockwood \& Wilkins Fractures in children. 5th edition. Philadelphia: Lippincott Willians \& Wilkins; 2001. p. 930-8.

11. Macnicol MF. The Scottish incidence of traumatic dislocation of the hip in childhood. J Pediatr Orthop B. 2000;9(2):122-4.

12. Price CT, Phillips JH, Devito DP. Management of fractures. In: Morrisy RT, Weinstein SL. Lovell \& Winter's pediatric orthopaedics. 5th edition. Philadelphia: Lippincott Willians \& Wilkins; 2001. p. 1372-3.

13. Price CT, Pyevich MT, Knapp DR, Phillips JH, Hawker JJ. Traumatic hip dislocation with spontaneous incomplete reduction: a diagnostic trap. J Orthop Trauma. 2002;16(10):730-5.

14. Banskota AK, Spiegel DA, Shrestha S, Shrestha OP, Rajbhandary T. Open reduction for neglected traumatic hip dislocation in children and adolescents. X-ray transparency interpositions after reduction of traumatic dislocations of the hip in children. J Pediatr Orthop. 2007;27(2):187-91.

15. Olsson O, Landin LA, Johansson A. Traumatic hip dislocation with spontaneous reduction and capsular interposition. A report of 2 cases. Acta Orthop Scand. 1994;65(4):476-9

16. Burgos J, Gonzales-Herrans P, Ocete G. Traumatic hip dislocation with incomplete reduction due to soft tissue interposition in a 4-year-old boy. J Pediatr Orthop B. 1995;4(2):216-8.

17. Rieger H, Pennig D, Klein W, Grunert J. Traumatic dislocation of the hip in young children. Arch Orthop Trauma Surg. 1991;110(2):114-7.

18. Mehlman CT, Hubbard GW, Crawford AH, Roy DR, Wall EJ. Traumatic hip dislocation in children. Long-term followup of 42 patients. Clin Orthop Relat Res. 2000;(376):68-79.

19. Pinheiro PCMS. Uma lesão traumática no quadril da criança. Relato de um caso. Rev Bras Ortop. 1994;29(1/2):44-6.

20. Salisbury RD, Eastwood DM. Traumatic dislocation of the hip in chlidren. Clin Orthop Relat Res. 2000;(377):106-11. 